DOI: $10.2478 /$ ausm-2014-0019

\title{
A particular Galois connection between relations and set functions
}

\author{
Árpád Száz \\ University of Debrecen \\ Department of Mathematics \\ H-4010 Debrecen, Pf. 12, Hungary \\ email: szaz@science.unideb.hu
}

\begin{abstract}
Motivated by a recent paper of U. Höhle and T. Kubiak on regular sup-preserving maps, we investigate a particular Galois-type connection between relations on one set $\mathrm{X}$ to another $\mathrm{Y}$ and functions on the power set $\mathcal{P}(\mathrm{X})$ to $\mathcal{P}(\mathrm{Y})$.

Since relations can largely be identified with union-preserving set functions, the results obtained can be used to provide some natural generalizations of most of the former results on relations and relators (families of relations). The results on inverses seem to be the only exceptions.
\end{abstract}

\section{Introduction}

In this paper, a subset $R$ of a product set $X \times Y$ is called a relation on $X$ to $Y$. And, a function $U$ on the power set $\mathcal{P}(X)$ to $\mathcal{P}(Y)$ is called a corelation on $\mathrm{X}$ to $\mathrm{Y}$.

Motivated by a recent paper of Höhle and Kubiak [9], for any relation $R$ on $X$ to $Y$, we define a correlation $R^{\star}$ on $X$ to $Y$ such that $R^{\star}(A)=R[A]$ for all $A \subset X$. Moreover, for any correlation $U$ on $X$ to $Y$, we define a relation $\mathrm{U}^{*}$ on $\mathrm{X}$ to $\mathrm{Y}$ such that $\mathrm{U}^{*}(\mathrm{x})=\mathrm{U}(\{\mathrm{x}\})$ for all $\mathrm{x} \in \mathrm{X}$.

And, we show that the functions $\star$ and $*$ establish an interesting Galoistype connection between the family $\mathcal{P}(X \times Y)$ of all relations on $X$ to $Y$ and the family $\mathcal{Q}(\mathrm{X}, \mathrm{Y})$ of all correlations on $\mathrm{X}$ to $\mathrm{Y}$, whenever $\mathcal{P}(\mathrm{X} \times \mathrm{Y})$ is 
considered to be partially ordered by the ordinary set inclusion and $\mathcal{Q}(\mathrm{X}, \mathrm{Y})$ by the pointwise one.

Since relations can largely be identified with union-preserving correlations, the results obtained can be used to provide some natural generalizations of most of the former results on relations and relators (families of relations). (The most relevant ones are in [21] and [16].) The results on inverse relations and relators seem to be the only exceptions.

To keep the paper almost completely self-contained, the most important definitions concerning relations, functions, ordered sets and Galois connections $[5$, p. 155$]$ will be briefly listed in the next two preparatory sections in somewhat novel forms. They will clarify our subsequent results and show the way to further investigations on Galois-type connections.

\section{Relations and functions}

A subset $\mathrm{F}$ of a product set $\mathrm{X} \times \mathrm{Y}$ is called a relation on $\mathrm{X}$ to $\mathrm{Y}$. If in particular $F \subset X^{2}$, with $X^{2}=X \times X$, then we may simply say that $F$ is a relation on $X$. In particular, $\Delta \mathrm{X}=\{(\mathrm{X}, \mathrm{X}): \mathrm{X} \in \mathrm{X}\}$ is called the identity relation on $\mathrm{X}$.

If $F$ is a relation on $X$ to $Y$, then for any $x \in X$ and $A \subset X$ the sets $F(x)=\{y \in Y:(x, y) \in F\}$ and $F[A]=\bigcup_{a \in A} F(a)$ are called the images of $x$ and $A$ under $F$ respectively. If $(x, y) \in F$, then we may also write $x F y$.

Moreover, the sets $D_{F}=\{x \in X: F(x) \neq \emptyset\}$ and $R_{F}=F[X]$ are called the domain and range of $\mathrm{F}$, respectively. If in particular $\mathrm{D}_{\mathrm{F}}=\mathrm{X}$, then we say that $\mathrm{F}$ is a relation of $\mathrm{X}$ to $\mathrm{Y}$, or that $\mathrm{F}$ is a non-partial relation on $\mathrm{X}$ to $\mathrm{Y}$.

In particular, a relation $f$ on $X$ to $Y$ is called a function if for each $x \in D_{f}$ there exists $y \in Y$ such that $f(x)=\{y\}$. In this case, by identifying singletons with their elements, we may simply write $f(x)=y$ in place of $f(x)=\{y\}$.

Moreover, a function $\star$ of $\mathrm{X}$ to itself is called a unary operation on $\mathrm{X}$. While, a function $*$ of $X^{2}$ to $X$ is called a binary operation on $X$. And, for any $x, y \in X$, we usually write $x^{\star}$ and $x * y$ instead of $\star(x)$ and $*((x, y))$.

For any relation $\mathrm{F}$ on $\mathrm{X}$ to $\mathrm{Y}$, we may naturally define a set-valued function $\mathrm{F}^{\diamond}$ on $\mathrm{X}$ such that $\mathrm{F}^{\diamond}(\mathrm{X})=\mathrm{F}(\mathrm{x})$ for all $\mathrm{x} \in \mathrm{X}$. This $\mathrm{F}^{\diamond}$ can be identified with $F$. However, thus in contrast to $F \subset X \times Y$ we already have $F^{\diamond} \subset X \times \mathcal{P}(Y)$.

Therefore, instead of $F^{\diamond}$, it is usually more convenient to work with $F$ or its selection functions. A function $f$ of $D_{F}$ to $Y$ is called a selection of $F$ if $f \subset F$, i.e., $f(x) \in F(x)$ for all $x \in D_{F}$.

Thus, the Axiom of Choice can be briefly expressed by saying that every relation has at least one selection function. Moreover, it can be easily seen 
that each relation is the union of its selection functions.

If $F$ is a relation on $X$ to $Y$, then $F=\bigcup_{x \in X}\{x\} \times F(x)$. Therefore, the values $F(x)$, where $x \in X$, uniquely determine $F$. Thus, a relation $F$ on $X$ to $Y$ can be naturally defined by specifying $F(x)$ for all $x \in X$.

For instance, the complement relation $\mathrm{F}^{\mathrm{c}}$ can be naturally defined such that $F^{c}(x)=F(x)^{c}=Y \backslash F(x)$ for all $x \in X$. The latter notation will not cause confusions, since thus we also have $F^{c}=X \times Y \backslash F$.

Quite similarly, the inverse relation $\mathrm{F}^{-1}$ can be naturally defined such that $F^{-1}(y)=\{x \in X: y \in F(x)\}$ for all $y \in Y$. Thus, the operations $c$ and -1 are compatible in the sense $\left(F^{c}\right)^{-1}=\left(F^{-1}\right)^{c}$.

Moreover, if in addition $\mathrm{G}$ is a relation on $\mathrm{Y}$ to $\mathrm{Z}$, then the composition relation $G \circ F$ can be naturally defined such that $(G \circ F)(x)=G[F(x)]$ for all $x \in X$. Thus, we also have $(G \circ F)[A]=G[F[A]]$ for all $A \subset X$.

On the other hand, if $\mathrm{G}$ is a relation on $\mathrm{Z}$ to $\mathrm{W}$, then the box product relation $\mathrm{F} \otimes \mathrm{G}$ can be naturally defined such that $(\mathrm{R} \otimes \mathrm{G})(x, z)=\mathrm{F}(\mathrm{x}) \times \mathrm{G}(z)$ for all $x \in X$ and $z \in Z$.

The box product relation, whose origin seems to go back to a thesis of J. Riquet in 1951, has been mainly investigated in [21]. In that, for instance, we have proved that $(F \otimes G)[A]=G \circ A \circ F^{-1}$ for all $A \subset X \times Z$.

Hence, by taking $A=\{(x, z)\}$, and $A=\Delta_{Y}$ if $Y=Z$, one can see that the box and composition products are actually equivalent tools. However, the box product can immediately be defined for an arbitrary family of relations.

\section{Generalized ordered sets and Galois connections}

Now, a relation $R$ on $X$ may be called reflexive if $\Delta_{X} \subset R$, and transitive if $\mathrm{R} \circ \mathrm{R} \subset \mathrm{R}$. Moreover, $\mathrm{R}$ may be called symmetric if $\mathrm{R}^{-1} \subset \mathrm{R}$, and antisymmetric if $\mathrm{R} \cap \mathrm{R}^{-1} \subset \Delta_{\mathrm{X}}$.

Thus, a reflexive and transitive (symmetric) transitive relation may be called a preorder (tolerance) relation. And, a symmetric (antisymmetric) preorder relation may be called an equivalence (partial order) relation.

For instance, for $A \subset X$, the Pervin relation $P_{A}=A^{2} \cup A^{c} \times X[18]$ is a preorder relation on $X$. While, for a pseudo-metric $d$ on $X$ and $r>0$, the surrounding $\mathrm{B}_{\mathrm{r}}^{\mathrm{d}}=\left\{(\mathrm{x}, \mathrm{y}) \in \mathrm{X}^{2}: \mathrm{d}(\mathrm{x}, \mathrm{y})<\mathrm{r}\right\}$ is a tolerance relation on $\mathrm{X}$.

Moreover, we may recall that if $\mathcal{A}$ is a partition of $\mathrm{X}$, i. e., a family of pairwise disjoint, nonvoid subsets of $\mathrm{X}$ such that $\mathrm{X}=\bigcup \mathcal{A}$, then $\mathrm{E}_{\mathcal{A}}=$ $\bigcup_{A \in \mathcal{A}} A^{2}$ is an equivalence relation on $X$, which can be identified with $\mathcal{A}$. 
According to [15], an ordered pair $X(\leq)=(X, \leq)$, consisting of a set $X$ and a relation $\leq$ on $X$, will be called a generalized ordered set, or an ordered set without axioms. And, we shall usually write $\mathrm{X}$ in place of $\mathrm{X}(\leq)$.

Now, a generalized ordered set $X(\leq)$ may, for instance, be called reflexive if the relation $\leq$ is reflexive. Moreover, the generalized ordered set $X^{\prime}\left(\leq^{\prime}\right)=$ $X\left(\leq^{-1}\right)$ may be called the dual of $X(\leq)$.

Having in mind the terminology of Birkhoff $[1$, p. 1], a generalized ordered set will be briefly called a goset. Moreover, a preordered (partially ordered) set will be called a proset (poset).

Thus, every set $X$ is a proset with the universal relation $X^{2}$. Moreover, $X$ is a poset with the identity relation $\Delta_{X}$. And every subfamily of the power set $\mathcal{P}(X)$ is a poset with the ordinary set inclusion $\subset$.

The usual definitions on posets can be naturally extended to gosets [15] (And also to relator spaces [14] which include formal context [7, p. 17] as an important particular case).

For instance, for any subset $A$ of a goset $X$, we may naturally define

$$
\begin{aligned}
& \operatorname{lb}(A)=\{x \in X: \quad \forall a \in A: \quad x \leq a\}, \\
& \operatorname{ub}(A)=\{x \in X: \quad \forall a \in A: \quad a \leq x\},
\end{aligned}
$$

and

$$
\begin{array}{ll}
\min (A)=A \cap \operatorname{lb}(A), & \max (A)=A \cap u b(A), \\
\inf (A)=\max (\operatorname{lb}(A)), & \sup (A)=\min (u b(A)) .
\end{array}
$$

Thus, for instance, min may be considered as a relation on $\mathcal{P}(X)$ to $X$, or as a function of $\mathcal{P}(X)$ of to itself. However, if $X$ is antisymmetric, then $\operatorname{card}(\min (A)) \leq 1$ for all $A \subset X$. Therefore, min is actually a function.

Now, a goset $X$ may, for instance, be naturally called inf-complete if $\inf (A) \neq$ $\emptyset$ for all $A \subset X$. In [3], as an obvious extension of [1, Theorem 3, p. 112], we have proved that thus "inf-complete" is also equivalent to "sup-complete".

However, it now more important to note that, for any two subsets $A$ and $B$ of a goset $X$, we also have

$$
\mathrm{lb}(\mathrm{A}) \subset^{\prime} \mathrm{B} \Longleftrightarrow \mathrm{B} \subset \mathrm{lb}(\mathrm{A}) \Longleftrightarrow \mathrm{A} \subset \mathrm{ub}(\mathrm{B}) .
$$

Therefore, the set-functions $\mathrm{lb}$ and $\mathrm{ub}$ form a Galois connection between the poset $\mathcal{P}(X)$ and its dual in the sense of [5, Definition 7.23], suggested by Schmidt's reformulation [12, p. 209] of Ore's Galois connexion [10].

Instead of Galois connections, it is usually more convenient to use residuated mappings of Blyth and Janowitz [2] in some modified and generalized forms suggested by the present author in $[19,17,24,22]$. 
However, now for a function $f$ of one goset $X$ to another $Y$ and a function $g$ of $Y$ to $X$, we shall say that

(1) $f$ and $g$ form an increasing upper Galois connection between $X$ and $Y$ if $f(x) \leq y$ implies $x \leq g(y)$ for all $x \in X$ and $y \in Y$,

(2) $f$ and $g$ form an increasing lower Galois connection between $X$ and $Y$ if $x \leq g(y)$ implies $f(x) \leq y$ for all $x \in X$ and $y \in Y$.

Now, if both (1) and (2) hold, then we may naturally say that the functions $f$ and $g$ form an increasing Galois connection between $\mathrm{X}$ and $\mathrm{Y}$. Important examples for Galois connections can be found in [6]. ( See also [13, 16, 4].)

In the theory of relator spaces, it has turned out that the increasing upper and lower Galois connections are actually particular cases of upper and lower semicontinuous pairs of relations [20].

Therefore, they can be naturally extended to relators between relator spaces [23]. For this, it is enough to study first these connections only for functions between power sets instead of those between gosets.

\section{Functions on one power set to another}

Definition 1 If $U$ is a function on one power set $\mathcal{P}(X)$ to another $\mathcal{P}(Y)$, then we simply say that $\mathrm{U}$ is a correlation on $\mathrm{X}$ to $\mathrm{Y}$.

Remark 1 According to Birkhoff [1, p. 111], the term "operation on X" could also be used. However, this may cause some confusions because of the customary meaning of this expression.

Definition 2 A correlation $\mathrm{U}$ on $\mathrm{X}$ to $\mathrm{Y}$, is called

(1) increasing if $U(A) \subset U(B)$ for all $A \subset B \subset X$,

(2) quasi-increasing if $\mathrm{U}(\{x\}) \subset \mathrm{U}(\mathrm{A})$ for all $x \in A \subset X$,

(3) union-preserving if $\mathrm{U}(\cup \mathcal{A})=\bigcup_{A \in \mathcal{A}} \mathrm{U}(\mathrm{A})$ for all $\mathcal{A} \subset \mathcal{P}(\mathrm{X})$.

Remark 2 In the $X=Y$ particular case, $U$ may also be naturally called extensive, intensive, involutiv, and idempotent if $A \subset \mathrm{U}(A), \mathrm{U}(\mathrm{A}) \subset \mathrm{A}$, $\mathrm{U}(\mathrm{U}(A))=A$, and $\mathrm{U}(\mathrm{U}(A))=\mathrm{U}(A)$ for all $A \subset X$, respectively.

Moreover, in particular an increasing and idempotent correlation may be called a projection or modification operation. And an extensive (intensive) projection operation may be called a closure (interior) operation.

Simple reformulations of properties (2) and (1) in Definition 1 give the following two theorems. 
Theorem 1 For a correlation $\mathrm{U}$ on $\mathrm{X}$ to $\mathrm{Y}$, the following assertions are equivalent:

(1) U is quasi-increasing,

(2) $\bigcup_{x \in A} U(\{x\}) \subset U(A)$ for all $A \subset X$.

Theorem 2 For a corelation $\mathrm{U}$ on $\mathrm{X}$ to $\mathrm{Y}$, the following assertions are equivalent:

(1) U is increasing,

(2) $\bigcup_{A \in \mathcal{A}} \mathrm{U}(\mathrm{A}) \subset \mathrm{U}(\cup \mathcal{A})$ for all $\mathcal{A} \subset \mathcal{P}(\mathrm{X})$,

(3) $\mathrm{U}(\mathrm{A}) \cup \mathrm{U}(\mathrm{B}) \subset \mathrm{U}(\mathrm{A} \cup \mathrm{B})$ for all $\mathrm{A}, \mathrm{B} \subset \mathrm{X}$.

Hence, it is clear that in particular we also have

Corollary $1 A$ correlation $\mathrm{U}$ on $\mathrm{X}$ to $\mathrm{Y}$ is union-preserving if and only if it is increasing and $\mathrm{U}(\cup \mathcal{A}) \subset \bigcup_{\mathcal{A} \in \mathcal{A}} \mathrm{U}(\mathrm{A})$ for all $\mathcal{A} \subset \mathcal{P}(\mathrm{X})$.

However, it now more important to note that we also have the following theorem which has also been proved, in a different way, by Pataki [11]

Theorem 3 For a correlation $\mathrm{U}$ on $\mathrm{X}$ to $\mathrm{Y}$, the following assertions are equivalent:

(1) $\mathrm{U}$ is uninon-preserving,

(2) $\mathrm{U}(\mathrm{A})=\bigcup_{x \in A} \mathrm{U}(\{x\})$ for all $\mathrm{A} \subset \mathrm{X}$.

Proof. Since $A=\bigcup_{x \in A}\{x\}$ for all $A \subset X$, it is clear that (1) implies (2).

On the other hand, if (2) holds, then we can note that $U$ is already increasing. Therefore, to obtain (1), by Corollary 1 , we need only prove that $\cup(\cup \mathcal{A})$ $\subset \bigcup_{A \in \mathcal{A}} \mathrm{U}(\mathrm{A})$ for every $\mathcal{A} \subset \mathcal{P}(\mathrm{X})$.

For this, note that if $\mathcal{A} \subset \mathcal{P}(X)$, then by (2) we have

$$
\mathrm{u}(\bigcup \mathcal{A})=\bigcup_{x \in \cup \mathcal{A}} \mathrm{u}(\{x\}) .
$$

Therefore, if $\mathrm{y} \in \mathrm{U}(\cup \mathcal{A})$, then there exists $x \in \cup \mathcal{A}$ such that $\mathrm{y} \in \mathrm{U}(\{x\})$. Thus, in particular there exists $A_{o} \in \mathcal{A}$ such that $x \in A_{o}$, and so $\{x\} \subset A_{0}$. Hence, by using the increasingness of $\mathrm{U}$, we can already infer that

$$
y \in \mathrm{U}(\{x\}) \subset \mathrm{u}\left(\mathrm{A}_{\mathrm{o}}\right) \subset \bigcup_{A \in \mathcal{A}} \mathrm{u}(\mathrm{A}) \text {. }
$$

Therefore, the required inclusion is also true.

From this theorem, by Theorem 1, it is clear that in particular we also have 
Corollary $2 A$ correlation $\mathrm{U}$ on $\mathrm{X}$ to $\mathrm{Y}$ is union-preserving if and only if it is quasi-increasing and $\mathrm{U}(\mathrm{A}) \subset \bigcup_{\mathrm{x} \in \mathrm{A}} \mathrm{U}(\{x\})$ for all $\mathrm{A} \subset \mathrm{X}$.

Definition 3 For any two correlations $\mathrm{U}$ and $\mathrm{V}$ on $\mathrm{X}$ to $\mathrm{Y}$, we write

$$
\mathrm{U} \leq \mathrm{V} \Longleftrightarrow \mathrm{U}(\mathrm{A}) \subset \mathrm{V}(\mathrm{A}) \text { for all } \mathrm{A} \subset \mathrm{X} .
$$

Remark 3 Note that if in particular $U \subset V$, then $U(A)=V(A)$ for all $A \in D_{u}$ and $U(A)=\emptyset \subset V(A)$ for all $A \subset X$ with $A \notin D_{u}$. Therefore, we have $\mathrm{U}(A) \subset \mathrm{V}(A)$ for all $A \subset X$, and thus $U \leq V$.

Theorem 4 With the inequality considered in Definition 3, the family $\mathcal{Q}(\mathrm{X}, \mathrm{Y})$ of all correlations on $\mathrm{X}$ to $\mathrm{Y}$, forms a complete poset.

Proof. It can be easily seen that if $\mathcal{U}$ is a family of correlations on $\mathrm{X}$ to $\mathrm{Y}$ and

$$
V(A)=\bigcup_{U \in \mathcal{U}} u(A)
$$

for all $A \subset X$, then $V \in \mathcal{Q}(X, Y)$ such that $V=\sup (\mathcal{U})$.

Therefore, $\mathcal{Q}(X, Y)$ is sup-complete, and hence it is also inf-complete.

Remark 4 Note that if in particular each member of $\mathcal{U}$ is increasing (quasiincreasing), then $\mathrm{V}$ is also increasing (quasi-increasing).

Therefore, with the inequality given in Definition 3 , the family $\mathcal{Q}_{1}(\mathrm{X}, \mathrm{Y})$ of all quasi-increasing correlations on $\mathrm{X}$ to $\mathrm{Y}$ is also a complete poset.

\section{A Galois connection between relations and corre- lations}

According to the corresponding definitions of Höhle and Kubiak [9], we may also naturally introduce the following

Definition 4 For any relation $R$ on $X$ to $Y$, we define a correlation $R^{\star}$ on $X$ to $Y$ such that

$$
R^{\star}(A)=R[A]
$$

for all $A \subset X$.

Conversely, for any correlation $\mathrm{U}$ on $\mathrm{X}$ to $\mathrm{Y}$, we define a relation $\mathrm{U}^{*}$ on $\mathrm{X}$ to $\mathrm{Y}$ such that

$$
\mathrm{U}^{*}(x)=\mathrm{U}(\{x\})
$$

for all $x \in X$. 
Now, by using the corresponding definitions, we can easily prove the following two theorems.

Theorem 5 If $\mathrm{U}$ is a correlation on $\mathrm{X}$ to $\mathrm{Y}$, then $\mathrm{R}^{\star} \leq \mathrm{U}$ implies $\mathrm{R} \subset \mathrm{U}^{*}$ for any relation $\mathrm{R}$ on $\mathrm{X}$ to $\mathrm{Y}$.

Proof. If $\mathrm{R}^{\star} \leq \mathrm{U}$, then by the corresponding definitions

$$
\mathrm{R}(\mathrm{x})=\mathrm{R}[\{x\}]=\mathrm{R}^{\star}(\{x\}) \subset \mathrm{U}(\{x\})=\mathrm{U}^{*}(x)
$$

for all $x \in X$. Therefore, $\mathrm{R} \subset \mathrm{U}^{*}$ also holds.

Theorem 6 For a correlation $\mathrm{U}$ on $\mathrm{X}$ to $\mathrm{Y}$, the following assertions are equivalent:

(1) U is quasi-increasing,

(2) $\mathrm{R} \subset \mathrm{U}^{*}$ implies $\mathrm{R}^{\star} \leq \mathrm{U}$ for any relation $\mathrm{R}$ on $\mathrm{X}$ to $\mathrm{Y}$.

Proof. If (1) holds and $\mathrm{R} \subset \mathrm{U}^{*}$, then

$$
\mathrm{R}^{\star}(\mathrm{A})=\mathrm{R}[\mathrm{A}]=\bigcup_{x \in A} \mathrm{R}(\mathrm{x}) \subset \bigcup_{x \in A} \mathrm{U}^{*}(x)=\bigcup_{x \in A} \mathrm{U}(\{x\}) \subset \mathrm{U}(\mathrm{A})
$$

for all $A \subset X$. Therefore, $R^{\star} \leq U$, and thus (2) also holds.

Conversely, if (2) holds, then because of $\mathrm{U}^{*} \subset \mathrm{U}^{*}$ we have $\left(\mathrm{U}^{*}\right)^{\star} \leq \mathrm{U}$. Therefore, for any $A \subset X$, we have

$$
\mathrm{U}^{* \star}(A) \subset \mathrm{U}(A) .
$$

Moreover, by using the corresponding definitions, we can see that

$$
\mathrm{U}^{* \star}(\mathrm{A})=\mathrm{U}^{*}[\mathrm{~A}]=\bigcup_{x \in A} \mathrm{u}^{*}(x)=\bigcup_{x \in A} \mathrm{u}(\{x\}) .
$$

Therefore, $\bigcup_{x \in A} \mathrm{U}(\{x\}) \subset \mathrm{U}(\mathrm{A})$, and thus (1) also holds.

Now, as an immediate consequence of the above two theorems, we can also state

Corollary 3 For an arbitrary relation $\mathrm{R}$ and a quasi-increasing correlation $\mathrm{U}$ on $\mathrm{X}$ to $\mathrm{Y}$, we have

$$
\mathrm{R}^{\star} \leq \mathrm{U} \quad \Longleftrightarrow \mathrm{R} \subset \mathrm{U}^{*} .
$$


Remark 5 This corollary shows that the operation $\star$ and the restriction of * to $\mathcal{Q}_{1}(\mathrm{X}, \mathrm{Y})$ establish an increasing Galois connection between the posets $\mathcal{P}(\mathrm{X} \times \mathrm{Y})$ and $\mathcal{Q}_{1}(\mathrm{X}, \mathrm{Y})$.

Therefore, the extensive theory of Galois connections ( see $[2,8,5]$ ) could be applied here. However, because of the simplicity of Definition 4, it seems now more convenient to use some elementary, direct proofs.

\section{Some further properties of the operations $\star$ and *}

By the corresponding definitions, we evidently have the following

Theorem 7 Under the notation of Definition 4,

(1) $\mathrm{R} \subset \mathrm{S}$ implies $\mathrm{R}^{\star} \leq \mathrm{S}^{\star}$ for any relations $\mathrm{R}$ and $\mathrm{S}$ on $\mathrm{X}$ to $\mathrm{Y}$,

(2) $\mathrm{U} \leq \mathrm{V}$ implies $\mathrm{U}^{*} \subset \mathrm{V}^{*}$ for any correlations $\mathrm{U}$ and $\mathrm{V}$ on $\mathrm{X}$ to $\mathrm{Y}$.

Remark 6 Note that, by using Corollary 3, instead of assertion (2), we could only prove that the restriction of the operation $*$ to $\mathcal{Q}_{1}(X, Y)$ is increasing.

From (2), by using Remark 3, we can immediately derive

Corollary $4 \mathrm{U} \subset \mathrm{V}$ also implies $\mathrm{U}^{*} \subset \mathrm{V}^{*}$ for any correlations $\mathrm{U}$ and $\mathrm{V}$ on $\mathrm{X}$ to $\mathrm{Y}$.

Moreover, we can also easily prove the following theorem whose first statement has also been established by Höhle and Kubiak [9] .

Theorem 8 For any two relations $\mathrm{R}$ and $\mathrm{S}$ on $\mathrm{X}$ to $\mathrm{Y}$,

(1) $R^{\star *}=R$,

(2) $\mathrm{R}^{\star} \leq \mathrm{S}^{\star}$ implies $\mathrm{R} \subset \mathrm{S}$.

Proof. By the corresponding definitions, we have

$$
\mathrm{R}^{\star *}(x)=\left(\mathrm{R}^{\star}\right)^{*}(x)=\mathrm{R}^{\star}(\{x\})=\mathrm{R}[\{x\}]=\mathrm{R}(x)
$$

for all $x \in X$. Therefore, (1) is also true.

To prove (2), note that if $R^{\star} \leq S^{\star}$ holds, then by Theorem 7 we also have $\mathrm{R}^{\star *} \subset \mathrm{S}^{\star *}$. Hence, by using (1), we can see that $\mathrm{R} \subset \mathrm{S}$ also holds. 
Remark 7 The above theorem shows that the function $\star$ is injective, $*$ is onto $\mathcal{P}(X, Y)$, and $\star *$ is the identity function of $\mathcal{P}(X \times Y)$.

Moreover, by Theorems 7 and 8 , we can also at once state

Corollary 5 For any two relations $\mathrm{R}$ and $\mathrm{S}$ on $\mathrm{X}$ to $\mathrm{Y}$, we have $\mathrm{R} \subset \mathrm{S}$ if and only if $\mathrm{R}^{\star} \leq \mathrm{S}^{\star}$.

Concerning the dual operation $* \star$, we can only prove the following theorem which, to some extent, has also been established by Höhle and Kubiak [9] and Pataki [11].

Theorem 9 For a corelation $\mathrm{U}$ on $\mathrm{X}$ to $\mathrm{Y}$, the following assertions are equivalent:

(1) $\mathrm{U}^{* \star}=\mathrm{U}$,

(2) $\mathrm{U}$ is union-preserving,

(3) $\mathrm{U}=\mathrm{R}^{\star}$ for some relation $\mathrm{R}$ on $\mathrm{X}$ to $\mathrm{Y}$.

Proof. If (2) holds, then by the proof of Theorem 6, and Theorem 3, we have

$$
\mathrm{U}^{* \star}(A)=\bigcup_{x \in A} \mathrm{u}(\{x\})=\mathrm{U}(\mathrm{A})
$$

for all $A \subset X$. Therefore, (1) also holds.

Now, since (1) trivially implies (3), we need only show that (3) also implies (2). For this, note that if (3) holds, then

$$
U(A)=R^{\star}(A)=R[A]=\bigcup_{x \in A} R(x)=\bigcup_{x \in A} R[\{x\}]=\bigcup_{x \in A} R^{\star}(\{x\})=\bigcup_{x \in A} U(\{x\})
$$

for all $\mathrm{A} \subset \mathrm{X}$. Therefore, by Theorem 3, assertion (2) also holds.

Remark 8 The above theorem shows that the function $\star$ maps $\mathcal{P}(X \times Y)$ onto the family $\mathcal{Q}_{3}(\mathrm{X}, \mathrm{Y})$ of all union-preserving correlations on $\mathrm{X}$ to $\mathrm{Y}$.

Moreover, the restriction of $*$ to $\mathcal{Q}_{3}(X, Y)$ is injective and that of $* \star$ is the identity function of $\mathcal{Q}_{3}(\mathrm{X}, \mathrm{Y})$. Therefore, the Galois connection mentioned in Remark 5 is rather particular.

Now, as an immediate consequence of Theorems 7 and 9 , we can also state 
Corollary 6 For any two union-preserving correlations $\mathrm{U}$ and $\mathrm{V}$ on $\mathrm{X}$ to $\mathrm{Y}$, we have $\mathrm{U} \leq \mathrm{V}$ if and only if $\mathrm{U}^{*} \subset \mathrm{V}^{*}$.

Proof. Note that if $\mathrm{U}^{*} \subset \mathrm{V}^{*}$ holds, then by Theorem 7 we also have $\mathrm{U}^{* \star} \leq$ $\mathrm{V}^{* \star}$. Hence, by Theorem 9 , we can see that $\mathrm{U} \leq \mathrm{V}$ also holds.

Moreover, in addition to Theorem 9, we can also prove the following

Theorem 10 Under the notation $\circ=* \star$, for any two correlations $\mathrm{U}$ and $\mathrm{V}$ on $\mathrm{X}$ to $\mathrm{Y}$, we have

(1) $\mathrm{U}^{\circ \circ}=\mathrm{U}^{\circ}$,

(2) $\mathrm{U} \leq \mathrm{V}$ implies $\mathrm{U}^{\circ} \leq \mathrm{V}^{\circ}$,

(3) $\mathrm{U}^{\circ} \leq \mathrm{U}$ if and only if $\mathrm{U}$ is quasi-increasing.

Proof. Assertion (2) is immediate from Theorem 7. While, from the proof of Theorem 6 , we know that

$$
\mathrm{u}^{\circ}(A)=\mathrm{U}^{* \star}(A)=\bigcup_{x \in A} \mathrm{u}(\{x\})
$$

for all $A \subset X$. Hence, by Definition 2 and Theorem 1, it is clear that (3) is true.

Moreover, from the above equality, we can also see that

$$
\mathrm{U}^{\circ}(A)=\bigcup_{x \in A} \mathrm{U}^{\circ}(\{x\})=\bigcup_{x \in A} \mathrm{u}(\{x\})=\mathrm{U}^{\circ}(A)
$$

for all $A \subset X$. Therefore, (1) is also true.

Remark 9 The above theorem shows that the function $\circ$ is a projection operation operation on $\mathcal{Q}(X, Y)$ such that its restriction to $\mathcal{Q}_{1}(\mathrm{X}, \mathrm{Y})$ is already an interior operation.

Moreover, from Theorem 9, we can see that, for any correlation $\mathrm{U}$ on $\mathrm{X}$ to $\mathrm{Y}$, we have $\mathrm{U}^{\circ}=\mathrm{U}$ if and only if $\mathrm{U}$ is union-preserving. Therefore, $\mathcal{Q}_{3}(\mathrm{X}, \mathrm{Y})$ is the family of all open elements of $\mathcal{Q}(\mathrm{X}, \mathrm{Y})$.

Now, as some useful consequences of our former results, we can also easily prove the following two theorems.

Theorem 11 If $\mathrm{R}$ is a relation on $\mathrm{X}$ to $\mathrm{Y}$ and $\mathrm{U}=\mathrm{R}^{\star}$, then

(1) $\mathrm{U}$ is the smallest quasi-increasing correlation on $\mathrm{X}$ to $\mathrm{Y}$ such that $\mathrm{R} \subset \mathrm{U}^{*}$,

(2) $\mathrm{U}$ is the largest union-preserving correlation on $\mathrm{X}$ to $\mathrm{Y}$ such that $\mathrm{U}^{*} \subset \mathrm{R}$. 
Proof. From Theorems 9 and 8 , we can see that $\mathrm{U}$ is union-preserving and $\mathrm{U}^{*}=\mathrm{R}^{\star *}=\mathrm{R}$.

Moreover, if $\mathrm{V}$ is a quasi-increasing corelation on $\mathrm{X}$ to $\mathrm{Y}$ such that $\mathrm{R} \subset \mathrm{V}^{*}$, then by Theorem 6 we also have $\mathrm{R}^{\star} \leq \mathrm{V}$, and thus $\mathrm{U} \leq \mathrm{V}$. Therefore, (1) is true.

On the other hand, if $\mathrm{V}$ is a correlation on $\mathrm{X}$ to $\mathrm{Y}$ such that $\mathrm{V}^{*} \subset \mathrm{R}$, then by Theorem 7 we also have $\mathrm{V}^{* \star} \leq \mathrm{R}^{\star}$, and thus $\mathrm{V}^{* \star} \leq \mathrm{U}$. Hence, if in particular $\mathrm{V}$ is union-preserving, then by Theorem 9 we can see that $\mathrm{V} \leq \mathrm{U}$. Therefore, (2) is also true.

Theorem 12 If $\mathrm{U}$ is a correlation on $\mathrm{X}$ to $\mathrm{Y}$ and $\mathrm{R}=\mathrm{U}^{*}$, then

(1) $\mathrm{R}$ is the largest relation on $\mathrm{X}$ to $\mathrm{Y}$ such that $\mathrm{R}^{\star} \leq \mathrm{U}$ whenever $\mathrm{U}$ is quasi-increasing,

(2) $\mathrm{R}$ is the smallest relation on $\mathrm{X}$ to $\mathrm{Y}$ such that $\mathrm{U} \leq \mathrm{R}^{\star}$ whenever $\mathrm{U}$ is union-preserving.

Proof. If $\mathrm{U}$ is quasi-increasing, then by Theorem 10 we have $\mathrm{R}^{\star}=\mathrm{U}^{* \star}=$ $\mathrm{U}^{\circ} \leq \mathrm{U}$. While, if $\mathrm{U}$ is union-preserving, then by Theorem 9 we have $\mathrm{R}^{\star}=$ $\mathrm{U}^{* \star}=\mathrm{U}$.

Moreover, if $\mathrm{S}$ is a relation on $\mathrm{X}$ to $\mathrm{Y}$ such that $\mathrm{S}^{\star} \leq \mathrm{U}$, then by Theorem 5 we also have $S \subset \mathrm{U}^{*}$, and thus $\mathrm{S} \subset \mathrm{R}$ even if $\mathrm{U}$ is not supposed to be quasi-increasing. Thus, in particular (1) is true.

While, if $S$ is a relation on $X$ to $Y$ such that $U \leq S^{\star}$, then by Theorem 7, we also have $\mathrm{U}^{*} \subset \mathrm{S}^{* *}$. Hence, by the definition of $\mathrm{R}$ and Theorem 8 , we can see that $\mathrm{R} \subset \mathrm{S}$ even if $\mathrm{U}$ is not supposed to be union-preserving. Thus, in particular (2) is also true.

Remark 10 Concerning the operations $\star$ and $*$, it is also worth noticing that if $\mathrm{R}$ is relation and $\mathrm{U}$ is a correlation on $\mathrm{X}$ to $\mathrm{Y}$, then by the corresponding definitions of [14] we have

(1) $R^{\star}(A)=\operatorname{cl}_{R^{-1}}(A)$ for all $A \subset X$,

(2) $R^{\star} \leq U \Longleftrightarrow A \in \operatorname{Int}_{R}(U(A))$ for all $A \subset X$.

Moreover, if $\mathrm{U}$ is quasi-increasing, then under the notation

$$
\operatorname{Int}_{\star}(\mathrm{U})=\left\{\mathrm{S} \subset \mathrm{X} \times \mathrm{Y}: \quad \mathrm{S}^{\star} \leq \mathrm{U}\right\}
$$

we have $\mathrm{U}^{*}=\max \left(\operatorname{Int}_{\star}(\mathrm{U})\right)=\bigcup \operatorname{Int}_{\star}(\mathrm{U})$ by assertion (1) in Theorem 12 . 


\section{Compatibility of the operation $\star$ with some set and relation theoretic ones}

Now, as some immediate consequence of the corresponding results on relations, we can also state the following theorems.

Theorem 13 If $\mathrm{R}$ is a relation on $\mathrm{X}$ to $\mathrm{Y}$, then for any family $\mathcal{A}$ of subsets of $\mathrm{X}$ we have

(1) $R^{\star}(\bigcup \mathcal{A})=\bigcup_{A \in \mathcal{A}} R^{\star}(A)$,

(2) $R^{\star}(\bigcap \mathcal{A}) \subset \bigcap_{A \in \mathcal{A}} R^{\star}(A)$.

Theorem 14 If $\mathrm{R}$ is a relation on $\mathrm{X}$ to $\mathrm{Y}$, then for any $\mathrm{A}, \mathrm{B} \subset \mathrm{X}$ we have

(1) $R^{\star}(A) \backslash R^{\star}(B) \subset R^{\star}(A \backslash B)$,

(2) $R^{\star}(A)^{c} \subset R^{\star}\left(A^{c}\right)$ if $Y=R[X]$.

Remark 11 If in particular $\mathrm{R}^{-1}$ is a function, then the corresponding equalities are also true in the above two theorems.

Theorem 15 If $\mathcal{R}$ is a family of relations on $\mathrm{X}$ to $\mathrm{Y}$, then for any $\mathrm{A} \subset \mathrm{X}$ we have

(1) $(\bigcup \mathcal{R})^{\star}(A)=\bigcup_{R \in \mathcal{R}} R^{\star}(A)$,

(2) $(\bigcap \mathcal{R})^{\star}(A) \subset \bigcap_{R \in \mathcal{R}} R^{\star}(A)$.

Theorem 16 If $\mathrm{R}$ and $\mathrm{S}$ are relations on $\mathrm{X}$ to $\mathrm{Y}$, then for any $\mathrm{A} \subset \mathrm{X}$ we have

(1) $R^{\star}(A) \backslash S^{\star}(A) \subset(R \backslash S)^{\star}(A), \quad(2) \quad R^{\star}(A)^{c} \subset R^{c \star}(A)$ if $A \neq \emptyset$.

Theorem 17 If $\mathrm{R}$ is a relation on $\mathrm{X}$ to $\mathrm{Y}$, then for any $\mathrm{A} \subset \mathrm{X}$ we have

$$
R^{c \star}(A)^{c}=\bigcap_{x \in A} R(x) .
$$

Moreover, we can also easily prove the following theorem which has also been established by Höhle and Kubiak [9] .

Theorem 18 For any two relations $\mathrm{R}$ on $\mathrm{X}$ to $\mathrm{Y}$ and $\mathrm{S}$ on $\mathrm{Y}$ to $\mathrm{Z}$, we have

$$
(S \circ R)^{\star}=S^{\star} \circ R^{\star} \text {. }
$$

Proof. By the corresponding definitions, we have

$$
(S \circ R)^{\star}(A)=(S \circ R)[A]=S[R[A]]=S^{\star}\left(R^{\star}(A)\right)=\left(S^{\star} \circ R^{\star}\right)(A)
$$

for all $A \subset X$. Therefore, the required equality is also true.

From this theorem, by using Theorem 9 , we can immediately derive 
Corollary 7 For an arbitrary relation on $\mathrm{R}$ on $\mathrm{X}$ to $\mathrm{Y}$ and a union-preserving correlation $\mathrm{V}$ on $\mathrm{Y}$ to $\mathrm{Z}$, we have

$$
\left(V^{*} \circ R\right)^{\star}=V \circ R^{\star}
$$

In addition to Theorem 18, we can also easily prove the following correction of a false statement of Höhle and Kubiak [9] .

Theorem 19 For an arbitrary correlation $\mathrm{U}$ on $\mathrm{X}$ to $\mathrm{Y}$ and a union-preserving correlation $\mathrm{V}$ on $\mathrm{Y}$ to $\mathrm{Z}$, we have

$$
(\mathrm{V} \circ \mathrm{U})^{*}=\mathrm{V}^{*} \circ \mathrm{U}^{*}
$$

Proof. By the corresponding definitions and Theorem 9, we have

$$
\begin{aligned}
(\mathrm{V} \circ \mathrm{U})^{*}(\mathrm{x}) & =(\mathrm{V} \circ \mathrm{U})(\{\mathrm{x}\})=\mathrm{V}(\mathrm{U}(\{\mathrm{x}\})) \\
& =\mathrm{V}\left(\mathrm{U}^{*}(\mathrm{x})\right)=\mathrm{V}^{* \star}\left(\mathrm{U}^{*}(\mathrm{x})\right)=\mathrm{V}^{*}\left[\mathrm{U}^{*}(\mathrm{x})\right]=\left(\mathrm{V}^{*} \circ \mathrm{U}^{*}\right)(\mathrm{x})
\end{aligned}
$$

for all $x \in X$. Therefore, the required equality is also true.

From this theorem, by using Theorems 9 and 8, we can immediately derive

Corollary 8 For a correlation $\mathrm{U}$ on $\mathrm{X}$ to $\mathrm{Y}$ and a relation $\mathrm{S}$ on $\mathrm{Y}$ to $\mathrm{Z}$, we have

$$
\left(\mathrm{S}^{\star} \circ \mathrm{U}\right)^{*}=\mathrm{S} \circ \mathrm{U}^{*}
$$

Remark 12 In addition to Theorem 18, it is also worth mentioning that if $\mathrm{R}$ is a relation on $\mathrm{X}$ to $\mathrm{Y}$ and $\mathrm{S}$ is a relation on $\mathrm{Z}$ to $\mathrm{W}$, then for any $\mathrm{A} \subset \mathrm{X} \times \mathrm{Z}$ we have

$$
(R \otimes S)^{\star}(A)=S \circ A \circ R^{-1} .
$$

\section{Partial compatibility of the operation $\star$ with the relation theoretic inversion}

Theorem 20 For a relation $\mathrm{R}$ on $\mathrm{X}$ to $\mathrm{Y}$, the following assertions are equivalent:

(1) $\mathrm{R}^{-1} \circ \mathrm{R}=\Delta_{\mathrm{X}}$

(2) $\left(\mathrm{R}^{\star}\right)^{-1} \subset\left(\mathrm{R}^{-1}\right)^{\star}$,

(3) $\mathrm{R}^{-1}$ is a function on $\mathrm{Y}$ onto $\mathrm{X}$. 
Proof. For any $x \in X$, we have

$$
\mathrm{R}^{\star}(\{x\})=\mathrm{R}[\{x\}]=\mathrm{R}(x), \quad \text { and thus } \quad\{x\} \in\left(\mathrm{R}^{\star}\right)^{-1}(\mathrm{R}(x)) .
$$

Hence, if (2) holds, we can infer that

$$
\{x\} \in\left(R^{-1}\right)^{\star}(R(x)), \quad \text { and thus } \quad\left(R^{-1}\right)^{\star}(R(x))=\{x\} .
$$

Therefore,

$$
R^{-1}[R(x)]=\{x\}, \quad \text { and thus } \quad\left(R^{-1} \circ R\right)(x)=\Delta_{X}(x) .
$$

Hence, we can see that (1) also holds.

To prove the converse implication, note that if $A \subset X$ and $B \subset Y$ such that $A \in\left(R^{\star}\right)^{-1}(B)$, then we also have

$$
R^{\star}(A)=B, \quad \text { and thus } \quad R[A]=B .
$$

Hence, we can infer that

$$
R^{-1}[R[A]]=R^{-1}[B], \quad \text { and thus }\left(R^{-1} \circ R\right)[A]=R^{-1}[B] .
$$

Therefore, if (1) holds, then

$$
\Delta_{X}[A]=R^{-1}[B], \quad \text { and thus } A=\left(R^{-1}\right)^{\star}(B) .
$$

Hence, it is clear that (2) also holds.

Therefore, (1) and (2) are equivalent. The proof of the equivalence of (1) and (3) will be left to the reader.

From Theorem 20, by writing $R^{-1}$ in place of $R$ we can immediately derive the following

Theorem 21 For a relation $\mathrm{R}$ on $\mathrm{X}$ to $\mathrm{Y}$, the following assertions are equivalent:

(1) $\mathrm{R} \circ \mathrm{R}^{-1}=\Delta_{\mathrm{Y}}$

(2) $\left(\mathrm{R}^{-1}\right)^{\star} \subset\left(\mathrm{R}^{\star}\right)^{-1}$,

(3) $\mathrm{R}$ is a function on $\mathrm{X}$ onto $\mathrm{Y}$.

Proof. Note that now $\mathrm{R}^{-1}$ is a relation on $\mathrm{Y}$ to $\mathrm{X}$. Therefore, by Theorem 20, the following assertions are equivalent:

(a) $\left(\mathrm{R}^{-1}\right)^{-1} \circ \mathrm{R}^{-1}=\Delta_{\mathrm{Y}}$; 
(b) $\left(\left(\mathrm{R}^{-1}\right)^{\star}\right)^{-1} \subset\left(\left(\mathrm{R}^{-1}\right)^{-1}\right)^{\star}$;

(c) $\left(R^{-1}\right)^{-1}$ is a function on $X$ onto $Y$.

Hence, since $R=\left(R^{-1}\right)^{-1}$, and

$$
\left(\mathrm{R}^{-1}\right)^{\star} \subset\left(\mathrm{R}^{\star}\right)^{-1} \Longleftrightarrow\left(\left(\mathrm{R}^{-1}\right)^{\star}\right)^{-1} \subset \mathrm{R}^{\star},
$$

it is clear that assertions (1), (2) and (3) are also equivalent.

Now, as an immediate consequence of the above two theorems, we can also state

Corollary 9 For a relation $\mathrm{R}$ on $\mathrm{X}$ to $\mathrm{Y}$, the following assertions are equivalent:

(1) $\left(R^{\star}\right)^{-1}=\left(R^{-1}\right)^{\star}$,

(2) $\mathrm{R}^{-1} \circ \mathrm{R}=\Delta_{\mathrm{X}}$ and $\mathrm{R} \circ \mathrm{R}^{-1}=\Delta_{\mathrm{Y}}$,

(3) $\mathrm{R}$ is an injective function of $\mathrm{X}$ onto $\mathrm{Y}$.

\section{Partial compatibility of the operation $*$ with the relation theoretic inversion}

From Theorem 20, by writing $\mathbf{U}^{*}$ in place of $\mathrm{R}$, we can easily derive

Theorem 22 If $\mathrm{U}$ is a union-preserving correlation on $\mathrm{X}$ to $\mathrm{Y}$ such that $\left(\mathrm{U}^{*}\right)^{-1}$ is a function on $\mathrm{Y}$ onto $\mathrm{X}$, then

$$
\left(\mathrm{u}^{-1}\right)^{*} \subset\left(\mathrm{u}^{*}\right)^{-1}
$$

Proof. Now, by Theorems and, we have

$$
\mathrm{u}^{-1}=\left(\mathrm{u}^{* \star}\right)^{-1}=\left(\left(\mathrm{u}^{*}\right)^{\star}\right)^{-1} \subset\left(\left(\mathrm{u}^{*}\right)^{-1}\right)^{\star} .
$$

Hence, by using Corollary and Theorem, we can infer that

$$
\left(\mathrm{u}^{-1}\right)^{*} \subset\left(\left(\left(\mathrm{u}^{*}\right)^{-1}\right)^{\star}\right)^{*}=\left(\left(\mathrm{u}^{*}\right)^{-1}\right)^{* *}=\left(\mathrm{u}^{*}\right)^{-1} .
$$

From Theorem 21, we can quite similarly derive the following 
Theorem 23 If $\mathrm{U}$ is a union-preserving correlation on $\mathrm{X}$ to $\mathrm{Y}$ such that $\mathrm{U}^{*}$ is a function on $\mathrm{X}$ onto $\mathrm{Y}$, then

$$
\left(\mathrm{u}^{*}\right)^{-1} \subset\left(\mathrm{u}^{-1}\right)^{*} \text {. }
$$

Now, as an immediate consequence of the above two theorems, we can also state

Corollary 10 If $\mathrm{U}$ is a union-preserving correlation on $\mathrm{X}$ to $\mathrm{Y}$ such that $\mathrm{U}^{*}$ is an injective function of $\mathrm{X}$ onto $\mathrm{Y}$, then

$$
\left(\mathrm{u}^{*}\right)^{-1}=\left(\mathrm{u}^{-1}\right)^{*} \text {. }
$$

Moreover, by using Corollary 9, we can also easily prove the following

Theorem 24 If $\mathrm{U}$ is an injective, union-preserving correlation on $\mathrm{X}$ to $\mathrm{Y}$ such that $\mathrm{U}^{-1}$ is also union-preserving, then the following assertions are equivalent:

(1) $\left(\mathrm{U}^{*}\right)^{-1}=\left(\mathrm{U}^{-1}\right)^{*}$,

(2) $\mathrm{U}^{*}$ is an injective function of $\mathrm{X}$ onto $\mathrm{Y}$.

Proof. Now, since the implication $(2) \Longrightarrow(1)$ has already been established in Corollary 10, we need only prove that (1) also implies (2).

For this note that if (1) holds, then by Theorem 9 we also have

$$
\left(\left(\mathrm{u}^{*}\right)^{\star}\right)^{-1}=\left(\mathrm{u}^{* \star}\right)^{-1}=\mathrm{u}^{-1}=\left(\mathrm{u}^{-1}\right)^{* \star}=\left(\left(\mathrm{u}^{-1}\right)^{*}\right)^{\star}=\left(\left(\mathrm{u}^{*}\right)^{-1}\right)^{\star} .
$$

Therefore, by Corollary 9, assertion (2) also holds.

From Corollary 9, we can also immediately derive the following

Theorem 25 For a symmetric relation $\mathrm{R}$ on $\mathrm{X}$, the following assertions are equivalent:

(1) $R^{2}=\Delta_{X}$,

(2) $\mathrm{R}^{\star}$ is an involution,

(3) $\mathrm{R}$ is an injective function of $\mathrm{X}$ onto $\mathrm{Y}$.

Remark 13 Moreover, by Theorem 18, we can at once see that, for an arbitrary relation $R$ on $X$, the correlation $R^{\star}$ is an involution if and only if $R^{2}=\Delta x$. That is, for any $x, y \in X$, we have $R(x) \cap R^{-1}(y) \neq \emptyset$ if and only if $x=y$. 


\section{Acknowledgement}

The work of the author has been supported by the Hungarian Scientific Research Fund (OTKA) Grant NK-81402.

The author is indebted to the referee who suggested shortening of the former preliminaries on relations and including of the relevant definitions on Galois connections.

\section{References}

[1] G. Birkhoff, Lattice theory, Amer. Math. Soc. Colloq. Publ. 25, Providence, Rhode Island, 1967.

[2] T.S. Blyth, M.F. Janowitz, Residuation theory, Pergamon Press, Oxford, 1972.

[3] Z. Boros, Á. Száz, Infimum and supremarkum completeness properties of ordered sets without axioms, An. Stiinţ. Univ. Ovidius Constanta Ser. Mat., 16 (2008), 1-7.

[4] S. Buglyó, Á. Száz, A more important Galois connection between distance functions and inequality relations, Sci. Ser. A Math. Sci. (N.S.), 18 (2009), 17-38.

[5] B. A. Davey, H. A. Priestley, Introduction to lattices and order, Cambridge University Press, Cambridge, 2002.

[6] K. Denecke, M. Erné, S. L. Wismath (Eds.) Galois connections and applications, Kluwer Academic Publisher, Dordrecht, 2004.

[7] B. Ganter, R. Wille, Formal concept analysis, Springer-Verlag, Berlin, 1999.

[8] G. Gierz, K. H. Hofmann, K. Keimel, J.D. Lawson, M. Mislove, D. S. Scott, A compendium of continuous lattices, Springer-Verlag, Berlin, 1980.

[9] U. Höhle, T. Kubiak, On regularity of sup-preserving maps: generalizing Zarecki1̌'s theoremark, Semigroup Forum, 83 (2011), 313-319.

[10] O. Ore, Galois connexions, Trans. Amer. Math. Soc., 55 (1944), 493-513.

[11] G. Pataki, On a generalized infimal convolution of set functions, Ann. Math. Sil., 27 (2013), 99-106. 
[12] J. Schmidt, Beiträge zur filtertheorie II, Math. Nachr., 10 (1953), 197-232.

[13] Á. Száz, A Galois connection between distance functions and inequality relations, Math. Bohem., 127 (2002), 437-448.

[14] Á. Száz, Upper and lower bounds in relator spaces, Serdica Math. J., 29 (2003), 239-270.

[15] Á. Száz, Lower and upper bounds in ordered sets without axioms, Tech. Rep., Inst. Math., Univ. Debrecen, 2004/1, 11 pp.

[16] Á. Száz, Galois-type connections on power sets and their applications to relators, Tech. Rep., Inst. Math., Univ. Debrecen, 2005/2, 38 pp.

[17] Á. Száz, Supremum properties of Galois-type connections, Comment. Math. Univ. Carolin., 47 (2006), 569-583.

[18] Á. Száz, Minimal structures, generalized topologies, and ascending systems should not be studied without generalized uniformities, Filomat, 21 (2007), 87-97.

[19] Á. Száz, Galois type connections and closure operations on preordered sets, Acta Math. Univ. Comenian., 78 (2009), 1-21.

[20] Á. Száz, Galois-type connections and continuities of pairs of relations, J. Int. Math. Virtual Inst., 2 (2012), 39-66.

[21] Á. Száz, Inclusions for composition and box products of relations, J. Int. Math. Virt. Inst., 3 (2013), 97-125.

[22] Á. Száz, Galois and Pataki connections revisited, Tech. Rep., Inst. Math., Univ. Debrecen, 2013/3, 20 pp.

[23] Á. Száz, Generalizations of Galois and Pataki connections to relator spaces, Tech. Rep., Inst. Math., Univ. Debrecen, 2014/1, 32 pp.

[24] Á. Száz, J. Túri, Comparisons and compositions of Galois-type connections, Miskolc Math. Notes, 7 (2006), 189-203. 